\title{
Revitalización de antiguos cuarteles a partir de un nuevo espacio climático. Criterios para la reducción de la demanda energética. Parque Científico Tecnológico Agroalimentario "PCiTAL". Gardeny. Lleida. Consorcio "PCiTAL"(Univ. Lérida- Ayuntamiento Lérida)
}

Revitalization of old headquarters from a new climatic space. Criteria for demand reduction. Food and Agriculture Technological Scientific Park "PCiTAL". Gardeny. Lleida. "PCiTAL" Consortium "PCiTAL" (Lérida University-Lérida City Hall)

T. Batlle $^{(*)}$, F. Pich-Aguilera ${ }^{(*)}$

\section{RESUMEN}

La rehabilitación-revitalización de unos antiguos cuarteles, situados en el área de Gardeny en la ciudad de Lérida, consistió en la intervención en tres edificios existentes y reconvertirlos en espacios de servicio a la investigación para la universidad y la empresa agroalimentaria. Se consideraron como prioridad, en las decisiones de proyecto, la mejora de la demanda energética de los edificios, para ello se utilizaron modelos informáticos, en la fase de proyecto, y monitorización, de los edificios existentes. Los datos y cálculos ajustaron las decisiones de proyecto y han sido la base para motorizar $y$ monitorizar el edificio finalizado. En fase de anteproyecto se evaluó mediante modelos energéticos la demanda energética asociada a las características arquitectónicas y constructivas del edificio para poder decidir las posibilidades de reducción. Las decisiones sobre los sistemas constructivos, los espacios-atrio y el efecto de humedad y vegetación nos permitían un ahorro en torno a un $23 \%$ en calefacción y un $50 \%$ en refrigeración.La posibilidad de integrar a un segundo grupo de investigación en el proyecto permitió por un lado cotejar los datos de ahorro y por otro monitorizar el edificio existente y así como programar la monitorización del edificio ya rehabilitado y en uso. Actualmente, sobre una metodología desarrollada, se están ajustando las estrategias bioclimáticas de control para dar el confort óptimo a los espacios intermedios e interiores de los edificios.

Palabras Clave: arquitectura-bioclimatismo-invernadero-demanda energética-control energético.

\section{SUMMARY}

The rehabilitation-revitalization of some old headquarters, situated in Gardeny area in the city of Lérida, consisted of the intervention in three existing buildings and there restructuring in investigation spaces for the university and the food and agriculture business. In project decisions, were considered to be a priority, the buildings energy demand improvement. For it, during the project phase, data processing models and storage of the existing building climatic information were used. The information and calculations fitted project and they have been the base to motorize and to monitor the rehabilitated building. In phase of preliminary design, and to the buildings energy demand improvement, the asoociated energy demand to the constructive and architectural characteristics of the building, were evaluated by means of energy models. The constructive systems solutions, atrium-spaces, and the humidity and vegetation effect, permitted savings around $23 \%$ in heating and $50 \%$ in cooling. The possibility to integrate a second research team in the project allowed us to arrange on one hand the data of savings and for other one monitoring the existing building. As well we programmed the rehabilitated (and in use) building monitoring. Nowadays, on a developed methodology, bioclimatic strategies are being adjusted to give the ideal comfort to the interiors and intermediate spaces of the buildings.

$113-93$

Keywords: architecture-bioclimatism-greenhouseenergy demands-energy control.
Informes de la Construcción Vol. 62, 517, 113-124, enero-marzo 2010 ISSN: 0020-0883 elSSN: 1988-3234 doi: 10.3989/ic.09.023

\footnotetext{
(*)Equipo Arquitectura Pich-Aguilera, Barcelona (España)

Persona de contacto/Corresponding author: teresa@picharchitects.com (T. Batlle)
} 


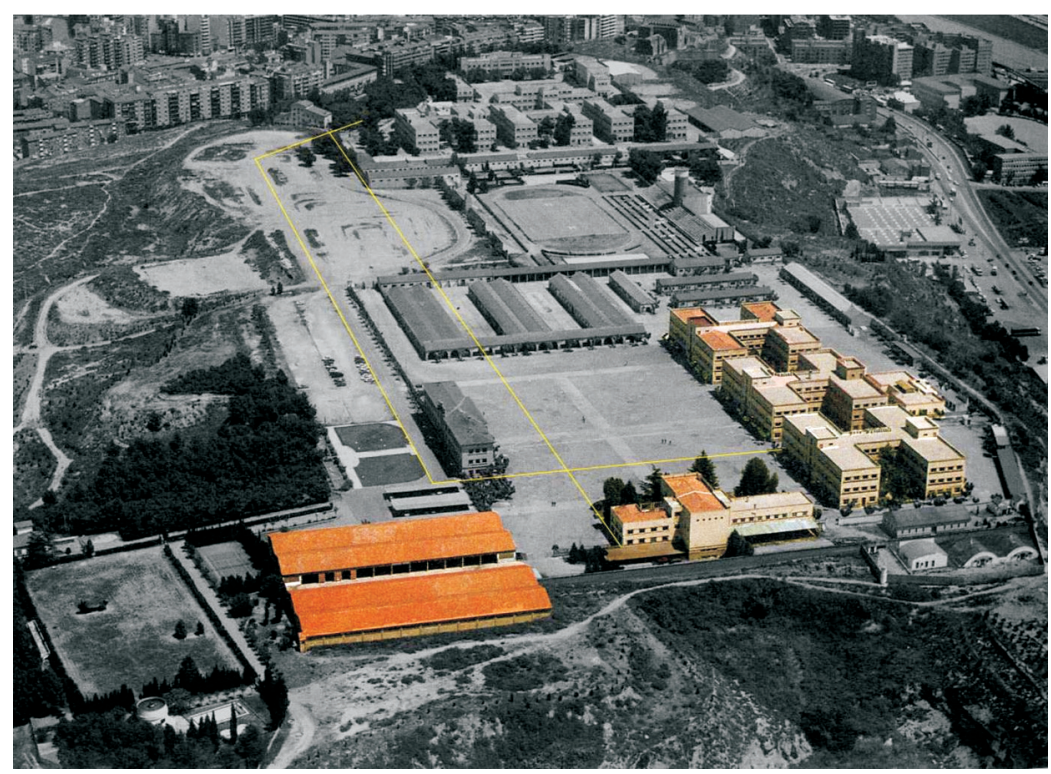

sostenibilidad. Tanto la apuesta por rehabilitar los edificios existentes, como la propuesta de proyecto de generar una nueva atmósfera que apoye el buen comportamiento bioclimático, hasta la decisión en los sistemas constructivos y de materiales, quisieron conseguir un respeto, principalmente ambiental, $y$, consecuentemente, una reducción de la demanda energética de los edificios.

Resumimos los aspectos principales, en este sentido, que se han desarrollado en el proyecto:

- Diseño bioclimático: protección y modulación de las ganancias térmicas, ventilación e iluminación natural, aprovechamiento de las condiciones del entorno del edificio.
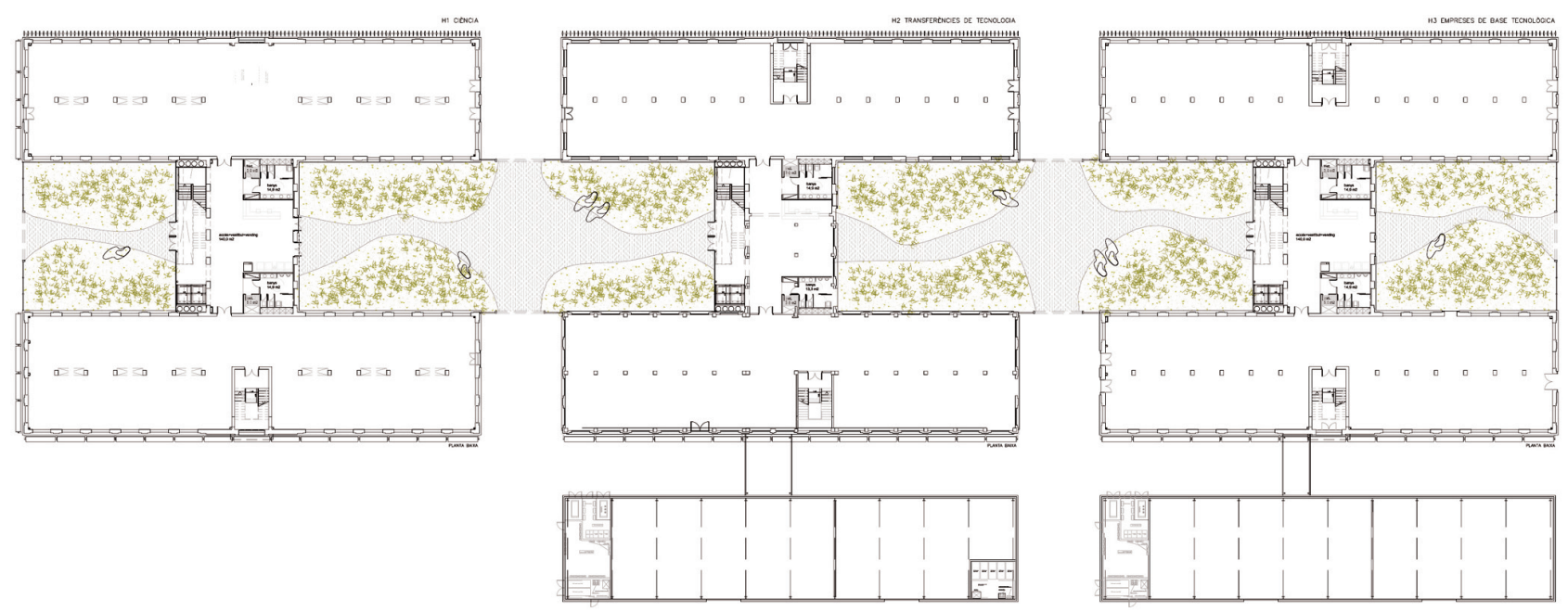

1. Visión de los cuarteles antes de su intervención.

2. Planta tipológica.
La rehabilitación-revitalización de unos antiguos cuarteles, situados en el área de Gardeny en la ciudad de Lérida, consistió en la intervención en tres edificios existentes y reconvertirlos en espacios de servicio a la investigación para la universidad y la empresa agroalimentaria.

La propuesta arquitectónica trató de reforzar la idea de los 6 volúmenes lineales existentes de planta diáfana, flexible y abierta a los cambios de uso y convertirlos en un único edificio, mediante la cobertura de los patios exteriores entre edificios, con unos invernaderos-umbráculos modulares, extraídos directamente del mundo innovador agrícola. Estos patios nos permitieron crear un espacio térmico, generador de un microclima, que apoya, desde sistemas pasivos de construcción, el confort interior de los edificios.

El proyecto parte de la voluntad de introducir, en la gestación y su proceso, criterios de
-Diseño de las instalaciones: control de la demanda energética. Integración de los sistemas de energías renovables. Eficiencia energética de las instalaciones.

-Elección materiales: utilización de materiales de bajo impacto ambiental y alta reciclabilidad. Gestión y separación selectiva de residuos, tanto en el proceso de construcción como en el uso del edificio. - Recursos: tratamiento de las aguas pluviales y de condensación. Limitación del consumo, desde mecanismos de ahorro de agua.

\section{DESCRIPCIÓN DE LOS ASPECTOS PRINCIPALES QUE PERMITEN UN BUEN COMPORTAMIENTO BIOCLIMÁTICO DE LOS EDIFICIOS}

\subsection{Espacio “invernadero-umbráculo"}

El proyecto plantea la cobertura de los patios centrales entre edificios con un "in- 
vernadero-umbráculo" industrializado -extraído directamente del reciclaje de invernaderos holandeses-, la obra y la normativa específica vigente española ha impedido el reciclaje del conjunto del invernadero, pero sí se ha podido reciclar la tecnología y algunos de los productos y sistemas que la industria aportaba.

\subsection{Descripcion constructiva invernadero- umbráculo}

El "invernadero-umbráculo" se cubre con vidrio y una estructura tubular metálica, en diente de sierra. Su configuración y detalles de materiales, remates y acabados extráe todo el conocimiento acumulado de captación térmica, recogida de aguas pluviales, recogida de aguas de condensación, sistemas monitorizados de aberturas de compuertas y sistemas de protección frente a la insolación.
- El cerramiento de los núcleos de comunicación vertical se resuelven con muro cortina y estructura de acero, utilizando el mismo sistema industrial de invernaderos.

\section{CUBIERTA DE LOS EDIFICIOS H EXISTENTES}

Todas las cubiertas han estado reformadas a lo largo del tiempo, en diferentes épocas y con diferentes tipos de soluciones. En fase de proyecto se analiza el estado de todas las cubiertas y se decide el derribo de todas las capas por encima del forjado de esta planta. Por encima de este nivel se coloca una cubierta ecológica aljibe.

\subsection{Descripción constructiva de las cubiertas}

-Cubierta ecológica aljibe, con gravas en las zonas de paso, sobre forjado existente.

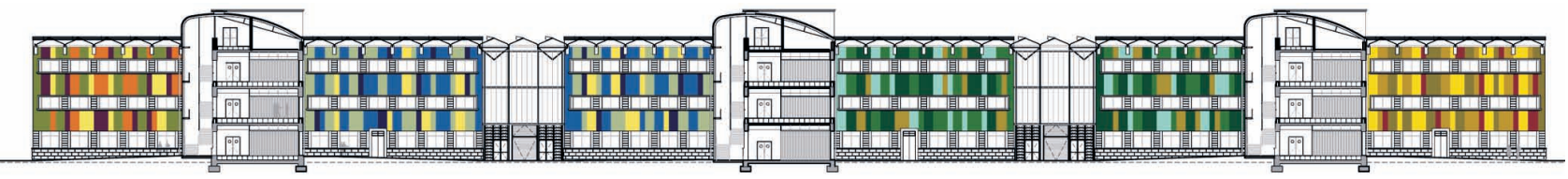

\section{CERRAMIENTOS-ENVOLVENTE EDIFICIOS H}

\subsection{Tratamientos de fachada}

Los edificios existentes no tenían aislamiento térmico, el punto prioritario estuvo en reforzar la inercia de los muros existentes.

Así pues en los muros exteriores se proyectó un aislamiento continuo desde el exterior, conservando la inercia térmica hacia el interior. En cambio se consideró positivo mantener sin aislamiento las fachadas en contacto con el "invernadero-umbráculo", de esta manera los muros irradian hacia el interior la temperatura de este espacio semiclimatizado.

\subsection{Descripción constructiva de las} intervenciones en fachadas existentes

- Tratamiento de las fachadas exteriores con aislamiento rebozado y pintado en las partes macizas (sistema sto).

- Tratamiento de las fachadas a patios, no se coloca aislamiento, únicamente se pintará con mortero mezclado con arlita.

- Se sustituyen las carpinterías exteriores; se proponen carpinterías de aluminio reciclado con rotura de puente térmico únicamente en las fachadas exteriores.

- Se protegen todas las aberturas exteriores con lamas. Existió en proyecto un análisis pormenorizado del tipo de lamas y su colocación según orientación que no pudo llevarse a cabo en todo su detalle.
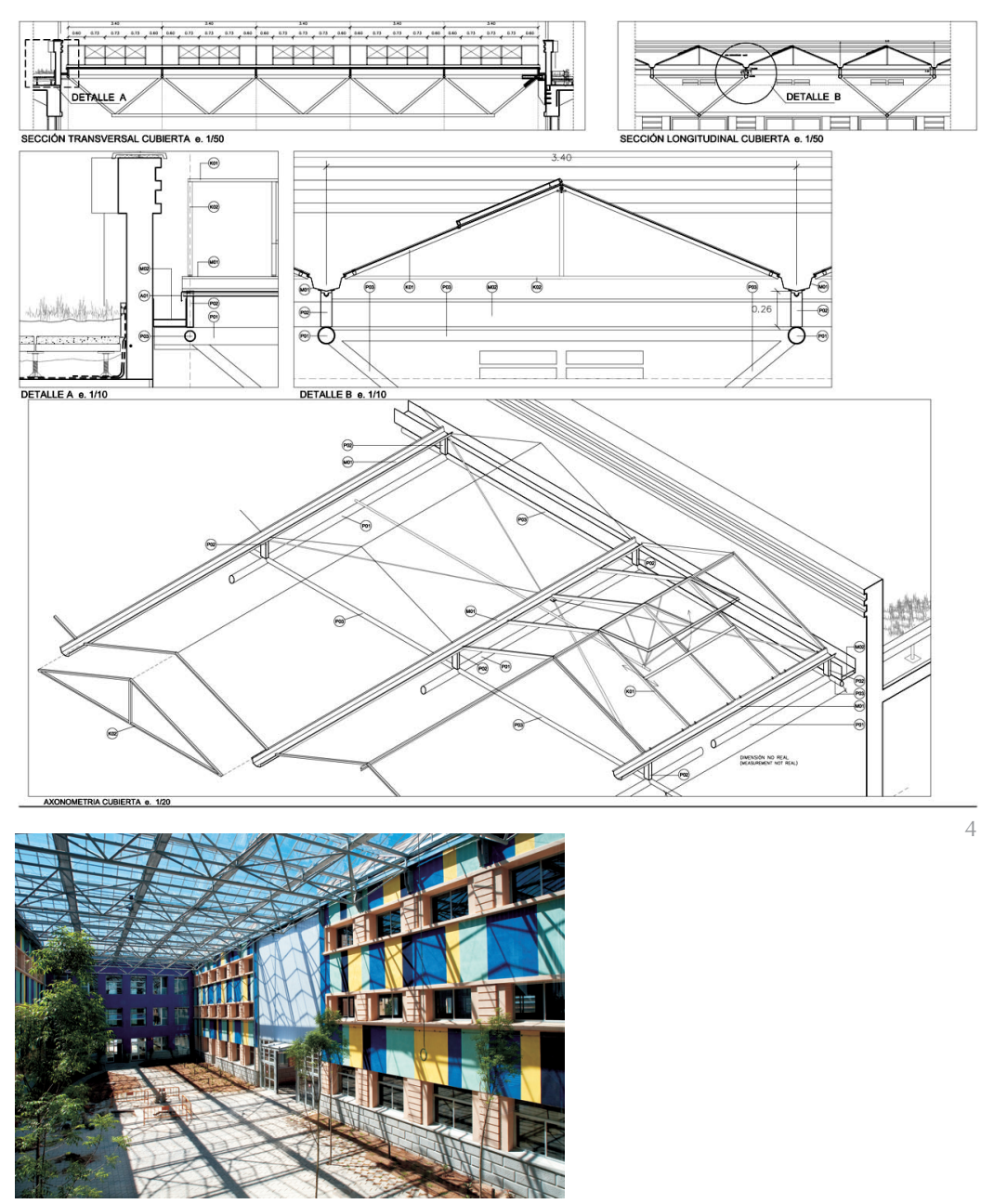


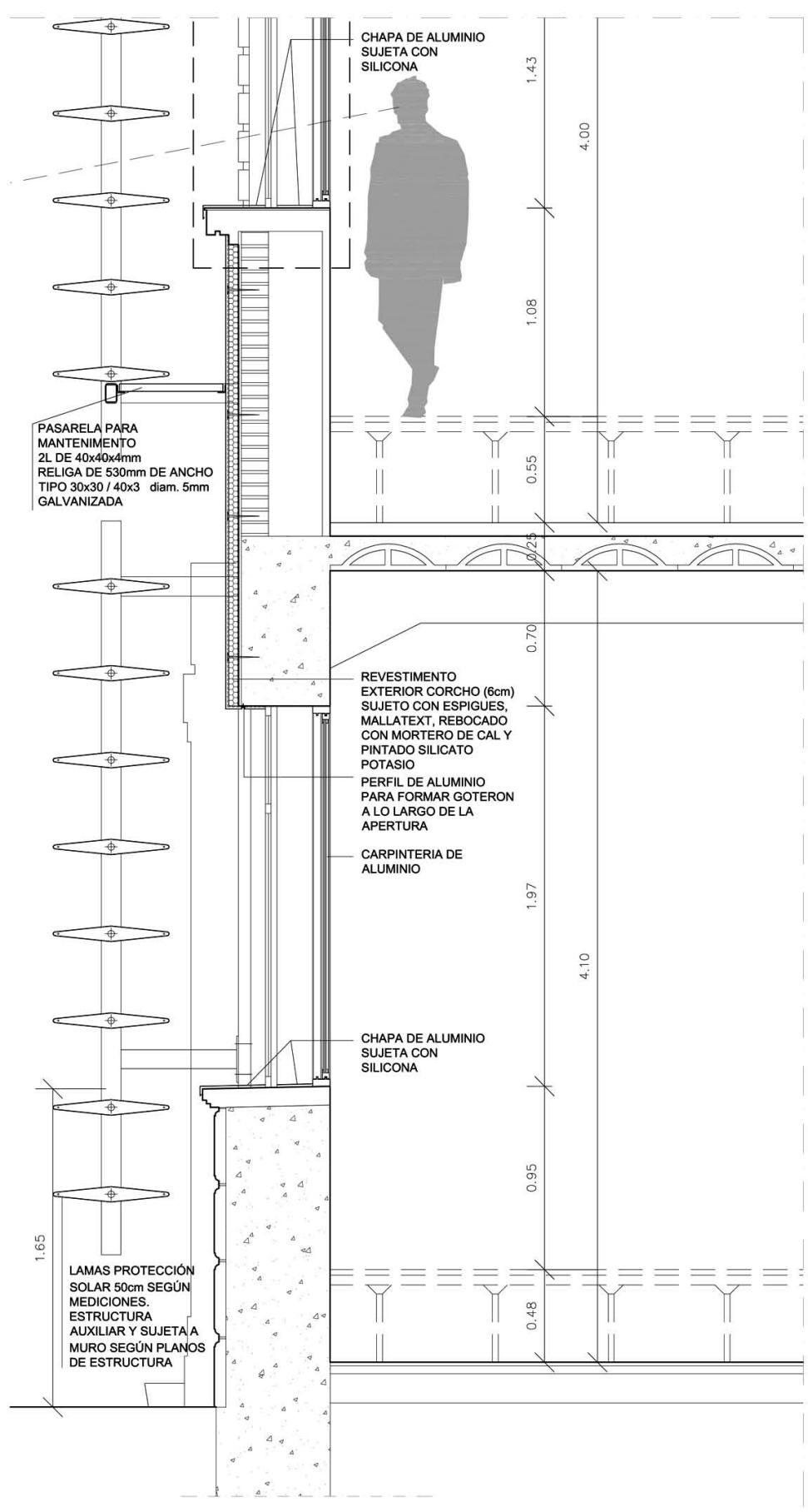

SECCIÓN FACHADA EXTERIOR rramientas informáticas y la contratación adicional de especialistas en el campo de la simulación de edificios para poder así cuantificar la reducción de la demanda energética en relación al ahorro conseguido y la inversión económica inicial que cada decisión suponía. Se colaboró, en la redacción del proyecto, con la asesoría ambiental Societat Orgánica.

\section{EVALUACIÓN DE LA DEMANDA ENERGÉTICA DE "PCiTAL" DESDE LAS HERRAMIENTAS ESTUDIADAS POR LA ASESORIA AMBIENTAL SOCIETAT ORGÁNICA}

La evaluación de la demanda energética se inició en fase de anteproyecto, así pues se evaluó mediante modelos energéticos la demanda energética asociada a las características arquitectónicas y constructivas del edificio y las posibilidades de reducción, considerando como premisa que tan sólo se puede actuar desde la envolvente.

El estudio se centra plenamente en el análisis de la demanda energética correspondiente a la climatización del edificio, entendiendo que habitualmente le corresponde el mayor porcentaje del consumo energético total, un 40 o un $60 \%$ del total de la demanda. Se consideró pues que el consumo asociado a otros usos energéticos estaba más ligado al uso y la gestión del edificio que a decisiones de proyecto.

Para evaluar la demanda energética de climatización asociada a las características del proyecto arquitectónico se tomó como base el anteproyecto trabajado y se introdujeron las herramientas seleccionadas. La información obtenida desde las herramientas de simulación en la fase de anteproyecto fueron contrastadas y analizadas en el proyecto básico y se revisaron nuevamente en el proyecto de ejecución. Los resultados se expresaron en términos de $\mathrm{kWh} / \mathrm{m}^{2}$ anuales de calefacción y refrigeración.

Se utilizaron dos herramientas informáticas:

Por un lado, en cumplimiento de la normativa, se empleó el apoyo de los datos que nos daba la herramienta de Código Técnico de la Edificación LIDER (Limitación de la Demanda Energética) ${ }^{(1)}$, en donde realizamos una simulación de ésta en régimen transitorio de transferencia de calor (considerando todas las zonas que tiene el edificio). El programa centra su cálculo en la envolvente del edificio con unos parámetros de confort prefijadas.

Por otro lado se utilizó la herramienta ECOTECT que nos permitió valorar de forma

odas las propuestas de proyecto fue cotejadas y validadas con el apoyo de he- 
Gráfico 1

Resultados de las demandas globales para el uso energético del edificio de cada una de las opciones en relación al edificio base.

\begin{tabular}{|c|c|c|c|c|c|c|c|c|c|c|c|}
\hline \multirow{2}{*}{\multicolumn{2}{|c|}{ Opción }} & \multicolumn{4}{|c|}{ SIMULACIÓN ECOTECT: Carga aparatos 60W/m² } & \multirow{3}{*}{$\begin{array}{l}\text { Total } \\
\text { estalvi }\end{array}$} & \multicolumn{4}{|c|}{ SIMULACIÓN ECOTECT: Carga aparatos 40W/m² } & \multirow{3}{*}{$\begin{array}{r}\text { Total } \\
\text { ahorro }\end{array}$} \\
\hline & & Calefacción & $\%$ ahorro & Refrigeración & $\%$ ahorro & & Calefacción & $\%$ ahorro & Refrigeración & $\%$ ahorro & \\
\hline 01 & Edificio base & $6,46 \mathrm{kWh} / \mathrm{m}^{2}$ & & $140,63 \mathrm{kWh} / \mathrm{m}^{2}$ & & & $9,55 \mathrm{kWh} / \mathrm{m}^{2}$ & & $125,14 \mathrm{kWh} / \mathrm{m}^{2}$ & & \\
\hline 02 & Base + aislamiento & $5,76 \mathrm{kWh} / \mathrm{m}^{2}$ & $11 \%$ & $136,32 \mathrm{kWh} / \mathrm{m}^{2}$ & $3 \%$ & $3 \%$ & $7,93 \mathrm{kWh} / \mathrm{m}^{2}$ & $17 \%$ & $103,94 \mathrm{kWh} / \mathrm{m}^{2}$ & $17 \%$ & $24 \%$ \\
\hline 03 & Base + atrios & $6,45 \mathrm{kWh} / \mathrm{m}^{2}$ & $0 \%$ & $120,70 \mathrm{kWh} / \mathrm{m}^{2}$ & $14 \%$ & $14 \%$ & $8,92 \mathrm{kWh} / \mathrm{m}^{2}$ & $7 \%$ & $88,58 \mathrm{kWh} / \mathrm{m}^{2}$ & $29 \%$ & $34 \%$ \\
\hline 04 & $\begin{array}{c}\text { Base }+ \text { atrios }+ \\
\text { mejoras (aislam, } \\
\text { lamas, cubiertas) }\end{array}$ & $5,19 \mathrm{kWh} / \mathrm{m}^{2}$ & $20 \%$ & $105,93 \mathrm{kWh} / \mathrm{m}^{2}$ & $25 \%$ & $24 \%$ & $5,97 \mathrm{kWh} / \mathrm{m}^{2}$ & $38 \%$ & $73,40 \mathrm{kWh} / \mathrm{m}^{2}$ & $41 \%$ & $46 \%$ \\
\hline 05 & $\begin{array}{c}\text { O4 + protección } \\
\text { atrios verano }\end{array}$ & $5,19 \mathrm{kWh} / \mathrm{m}^{2}$ & $20 \%$ & $102,98 \mathrm{kWh} / \mathrm{m}^{2}$ & $27 \%$ & $26 \%$ & $5,97 \mathrm{kWh} / \mathrm{m}^{2}$ & $38 \%$ & $66,34 \mathrm{kWh} / \mathrm{m}^{2}$ & $47 \%$ & $51 \%$ \\
\hline 06 & $\begin{array}{l}\text { O4 + efecto } \\
\text { vegetación }\end{array}$ & $5,19 \mathrm{kWh} / \mathrm{m}^{2}$ & $20 \%$ & $104,18 \mathrm{kWh} / \mathrm{m}^{2}$ & $26 \%$ & $26 \%$ & $5,97 \mathrm{kWh} / \mathrm{m}^{2}$ & $38 \%$ & $71,52 \mathrm{kWh} / \mathrm{m}^{2}$ & $43 \%$ & $47 \%$ \\
\hline
\end{tabular}

aproximada la incidencia de los diferentes factores que condicionan la demanda y adicionalmente permiten intervenir al usuario en muchos más parámetros que la herramienta LIDER, tales como los perfiles de ocupación, la utilización de los aparatos, así como un análisis detallado del asoleo y su incidencia en el interior del edificio. La herramienta ECOTECT nos permitió realizar un mayor número de simulaciones que con la herramienta LIDER (hemos de tener en cuenta que durante el desarrollo del proyecto el CTE no era de obligado cumplimiento y que se trabajó con la version beta del programa LIDER). Los parámetros más importantes de partida fueron la geometría del edificio y las soluciones constructivas propuestas, se contempló, tal como ya hemos descrito anteriormente, que la decisión de cobertura de los patios convirtiéndolos en atrio podían considerar el conjunto como un único edificio.

\section{Así pues se simularon 4 opciones:}

Opción 1: un edificio base, sin ninguna propuesta de mejora.

Opción 2: el edificio base con un aislamiento de $5 \mathrm{~cm}$ de lana de roca en fachadas exteriores.

Opción 3: el edificio base con la propuesta de atrios.

Opción 4: el edificio con todas las aportaciones de proyecto, refuerzo selectivo del aislamiento, cubierta ajardinada y lamas. Opción 5: protección de atrios en verano. Opción 6: efecto de la vegetación de hoja caduca.

Los parámetros principales para la simulación con la herramienta LIDER fueron: temperatura de confort en invierno de $20^{\circ}$, en verano $25^{\circ}$, cargas internas de $15 \mathrm{~W} / \mathrm{m}^{2}$,

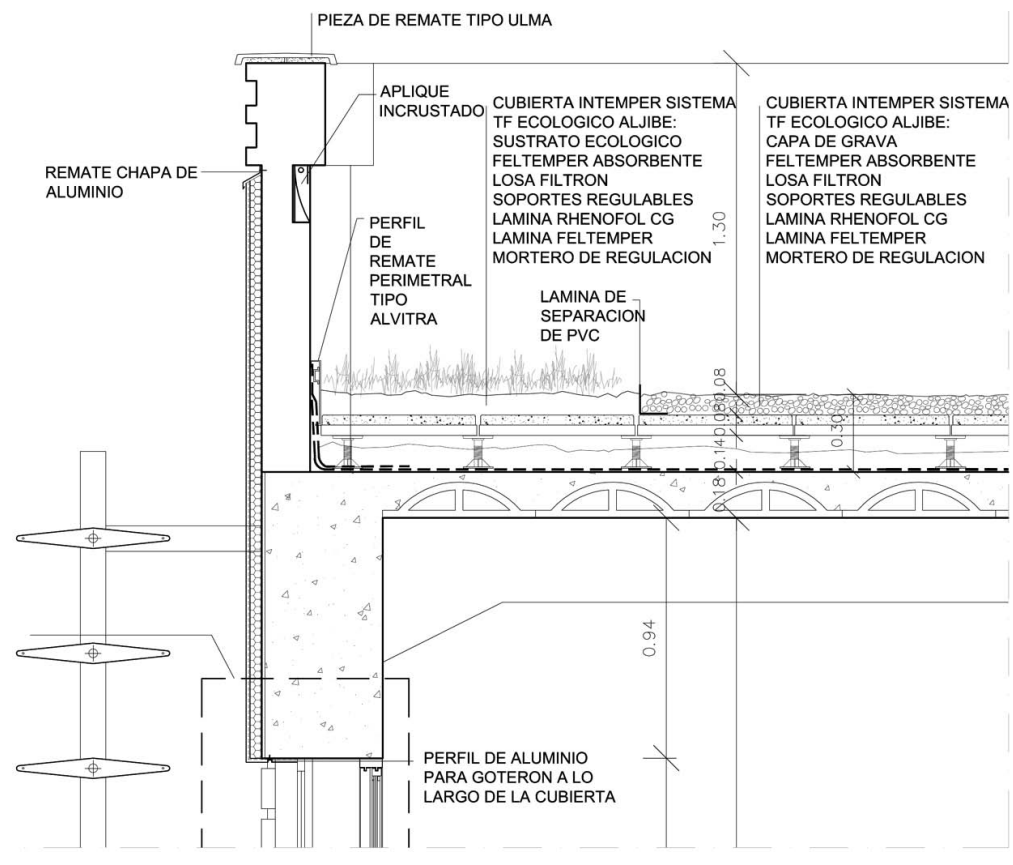

ocupación $10 \mathrm{~m}^{2} /$ persona. LIDER nos permitió definir cada una de las soluciones constructivas que se incorporaron en el proyecto y establecer si se trata de un edifico de baja o alta carga interna.

ECOTECT ${ }^{(2)}$ nos aportó la profundización de los parámetros de análisis y un mayor detalle en las opciones de simulación.

Finalmente los resultados de la demandas globales y por uso energético obtenidos con la herramienta ECOTECT para cada una de la opciones, en relación con un edificio base, se presentan en la siguiente tabla (incluye dos grupos de resultados para cada una de las alternativas de carga interna y una columna con el porcentaje de ahorro que se consigue en cada caso). Los resultados permiten verificar y detallar las tendencias observadas con la herramienta LIDER y justifican la alternativa de los atrios como espacios que
7. Detalle de las cubiertas aljibe ecológicas.

${ }^{1}$ Lider es una aplicación informática de calculo de la demanda energética establecida en el Documento Básico de Habitabilidad y Energía del Código Técnico de la Edificación, ofrecida por el Ministerio de la Vivienda y por el IDEA.

${ }^{2}$ El programa Ecotect permite medir y planificar el impacto ambiental del edificio diseñado, basado en un modelo tridimensional del edificio y todos los estudios posibles, asoleamiento, sombras, reflexiones de luz, ganancia de calor, ajustadas a lugar, país, épocas del año y clima. 


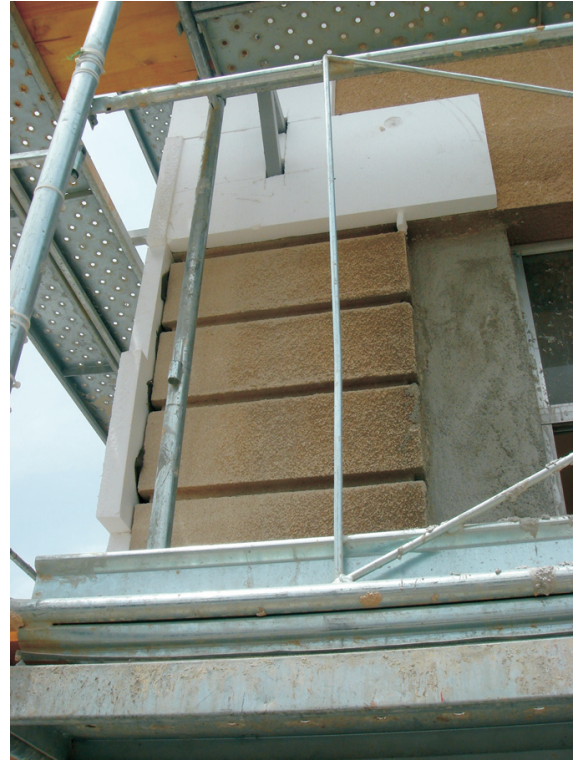

8
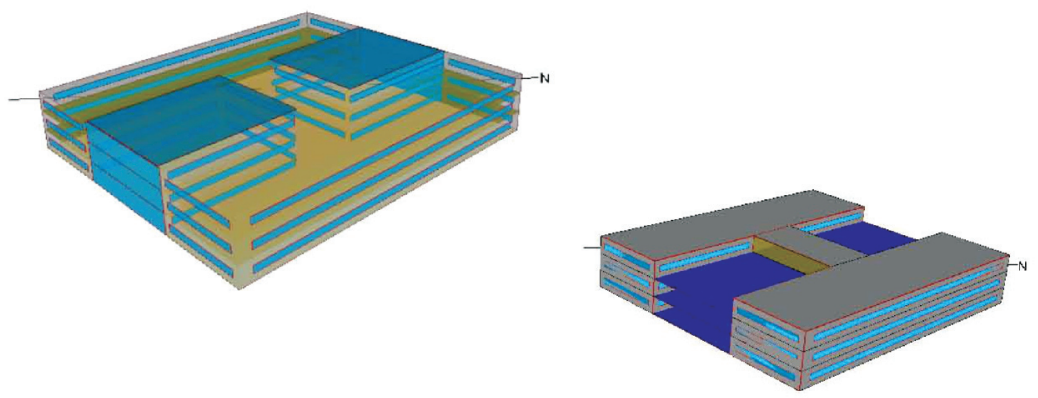

10
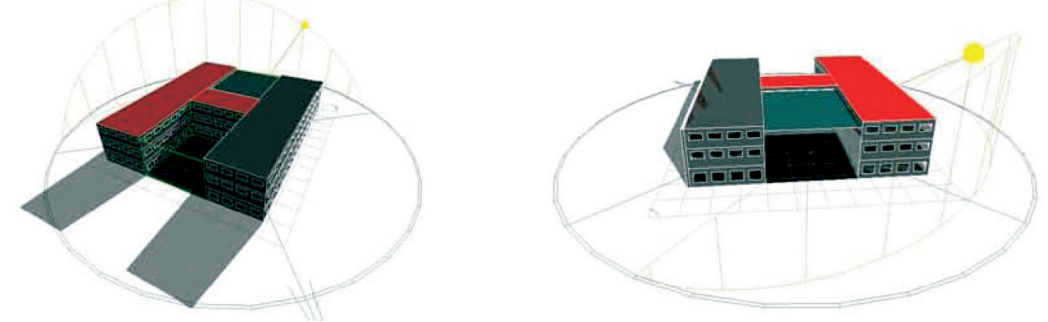

8. Detalle ejecución del aislamiento exterior.

9. Fotografía fachadas exteriores del edificio.

10. Modelización de las opciones tomadas en proyecto (Edificio con y si atrios) con LIDER.

11. Opciones de simulación con ECOTEC.

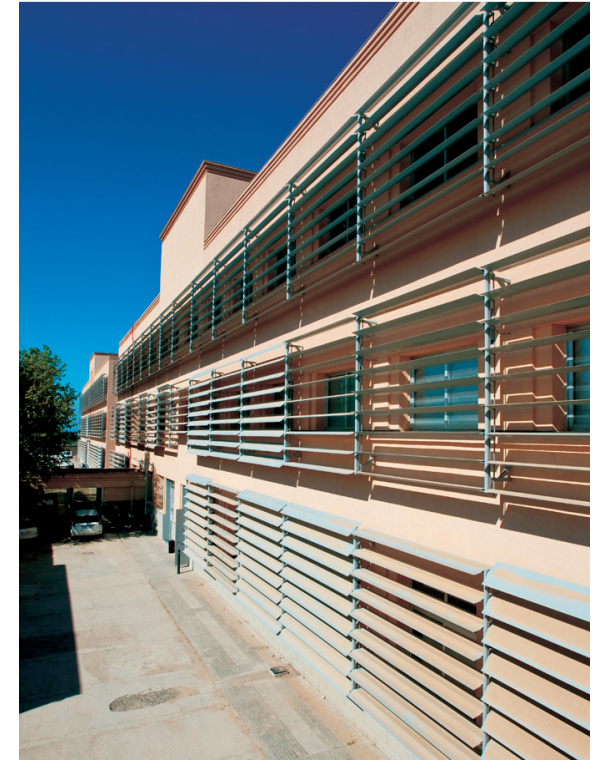

tanto en la fase anterior como posterior a la rehabilitación. Detallamos sintética y gráficamente el comportamiento bioclimático esperado, en invierno, verano y en relación con el uso del agua.

\section{SISTEMAS DE MONITORIZACIÓN DE LOS EDIFICIOS EXISTENTES. ESTUDIO REALIZADO POR EL NSTITUTO TECNOLÓGICO DE LÉRIDA, EL CENTRO TECNOLÓGICO BeeGroup-CIMNE Y EL GRUPO DE ENERGÍA SOLAR DE LA UNIVERSIDAD DE LÉRIDA}

El Instituto tecnológico de la Construcción de Lérida (ITL), juntamente con el Centro BeeGroup-CIMNE y el grupo de energía solar de la universidad de Lérida, plantearon un sistema de monitorización del comportamiento energético y el impacto de la rehabilitación de uno de los edificios existentes.

El objetivo prioritario del estudio liderado por BeeGroup-Cimne estaba, por un lado, en testear cuanto se había estudiado y concluido en proyecto $y$, por otro, profundizar en el actual comportamiento del edificio en aras a poder monitorizar y motorizar el edificio en uso una vez rehabilitado.

\section{PROCEDIMIENTO Y HERRAMIENTAS UTILIZADAS}

Se analizó el comportamiento energético de unas oficinas en uso, antes de la rehabilitación, mediante:

1- La inspección del edificio y sus instalaciones, juntamente con un periodo corto de monitorización. 


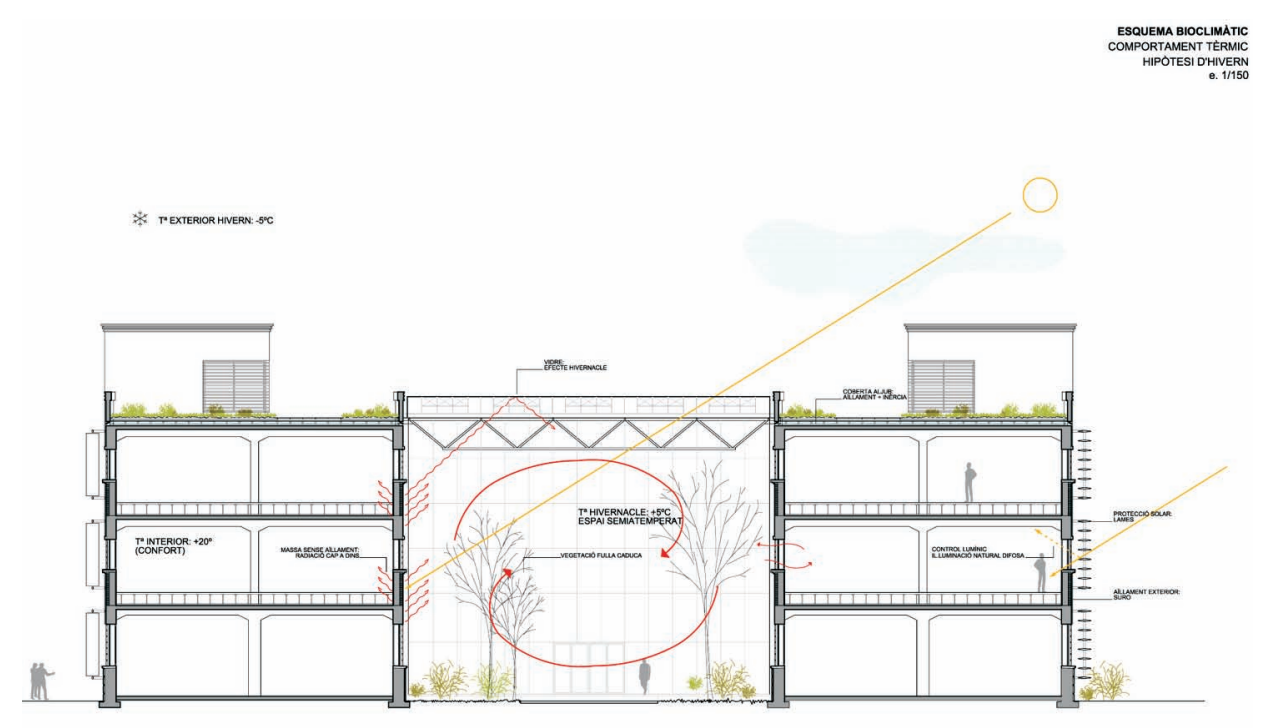

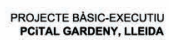

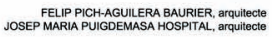

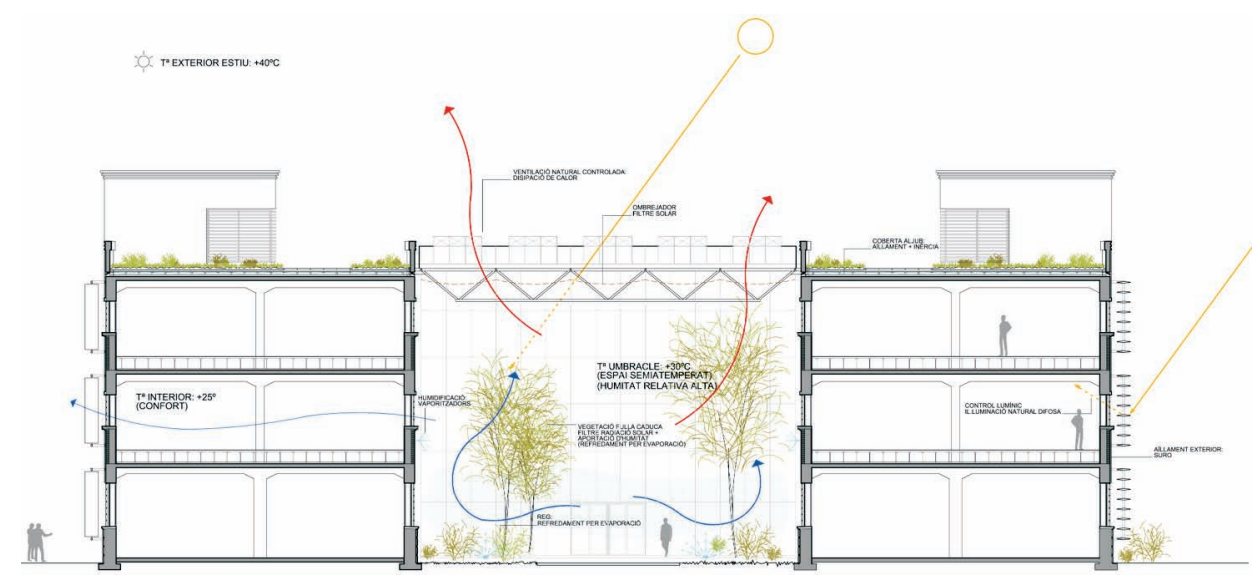

13

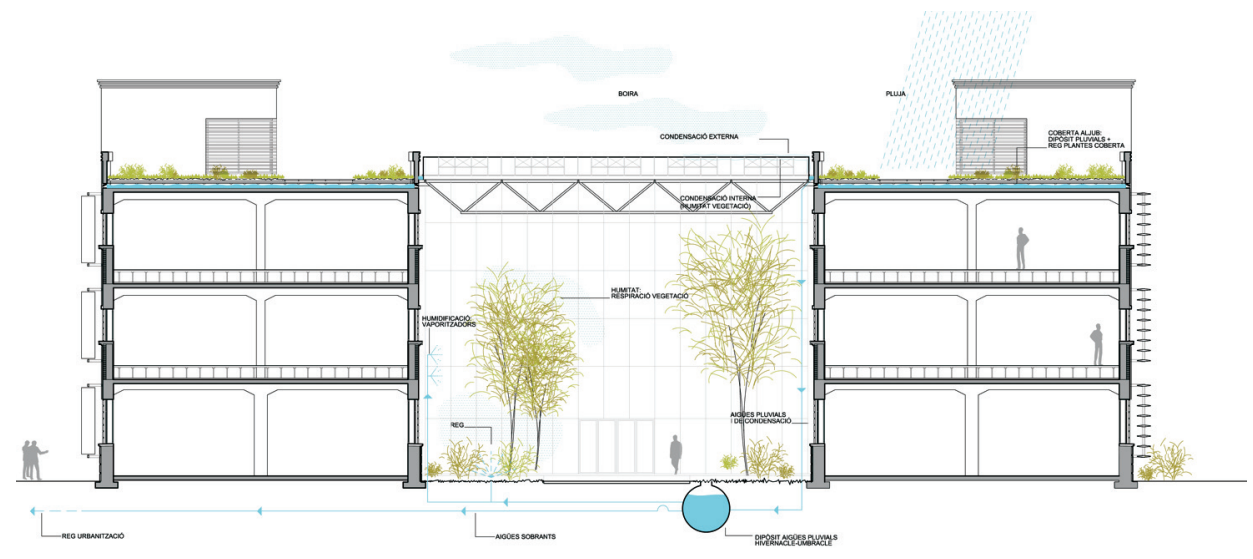

2- La Simulación del edificio con un simulador dinámico: Energy Plus( ${ }^{(3)}$ y Design Builder(4).

3- La simulación de las alternativas constructivas que se querían llevar a cabo en la remodelación del edificio.
12. Síntesis gráfica del comportamiento bioclimático en invierno.

13. Síntesis gráfica del comportamiento bioclimático en verano

14. Síntesis gráfica de la racionalización del agua de lluvia y de condensación.
14

${ }^{3}$ Energy Plus es un programa de simulación térmica y energética de edificios desarrollado por DOE (Department of Energy, Estados Unidos) con el que se pueden hacer estudios de demanda y consumo energético.

4- El estudio de desarrollo para disponer de un sistema comercial de control y gestión energética remota del proyecto definitivo.

Se estudió concretamente la primera planta a Sur, que corresponde a las dependencias del "Instituto Tecnológico de Lerida" (ITL).
${ }^{4}$ Design Builder permite obtener información precisa del comportamiento térmico del edificio y su renderizado en imagen y video en cualquier etapa del proceso de diseño. 
Gráfico 2

Esquema de monitorización y auditoría energética de las oficinas ITL (esquema estudio).

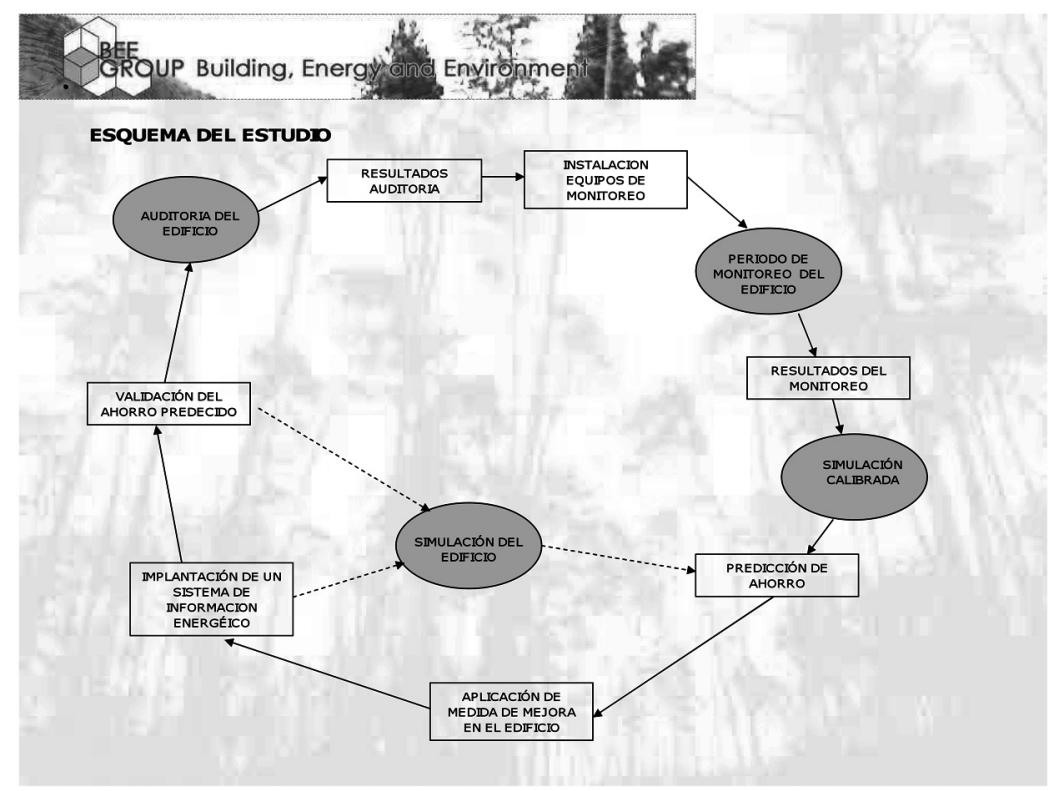

Gráfico 3

Espacio del edificio monitorizado.

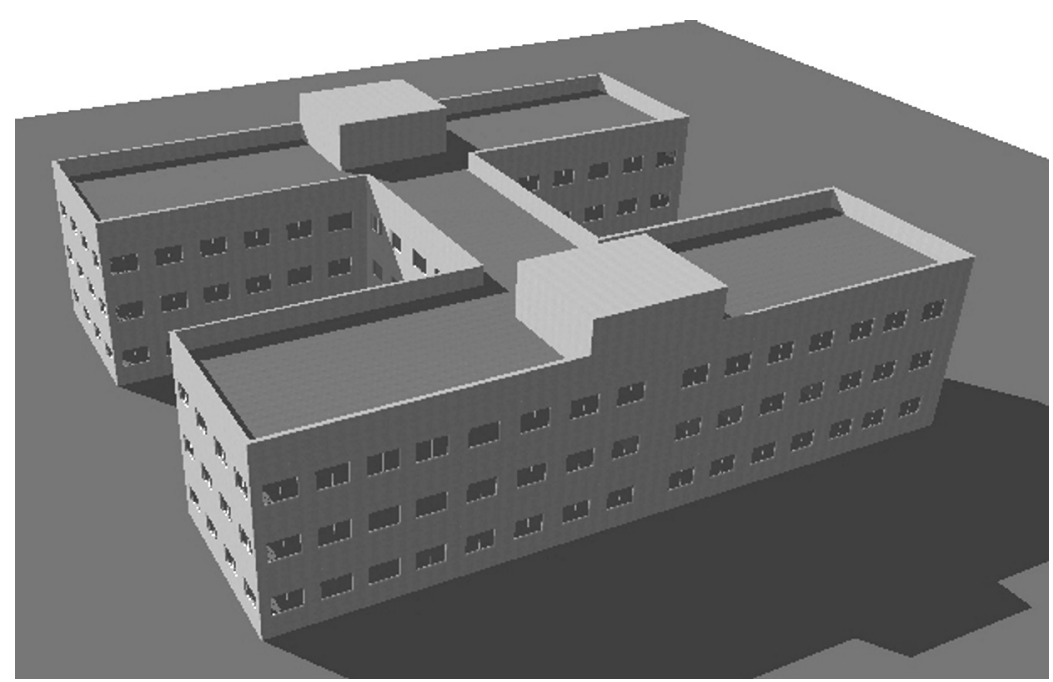

Los parámetros utilizados en la simulación fueron:

1. Geometría (localización, superficie y asoleo)

2. Muro exterior (el valor $\left(\mathrm{W} / \mathrm{m}^{2}-\mathrm{K}\right)$ de cada superficie del muro))

3. Particiones interiores (el valor $\left(\mathrm{W} / \mathrm{m}^{2}-\mathrm{K}\right)$ de todas las particiones interiores))

4. Ventanas (su tamaño y composición)

5. Ocupación (Número de personas y sus horarios)

6. Iluminación (tipos $\mathrm{W} / \mathrm{m}^{2}$ por luminaria)

7. Equipos (W/m² por equipo informático)

8. Climatización y Calefacción. (En una primera fase de estudio se ha analizado el edificio sin pensar en el apoyo de climatización).
Los datos climáticos fueron la extrapolación de un año tipo, siendo el año tipo el valor medio diario a lo largo de los últimos 20 años (hora a hora) y los datos obtenidos por una estación meteorológica, desde un programa informático específico que podía informar en detalle de la radiación solar.

Tal como hemos citado, para realizar la simulación energética del edificio se trabajó con el software Energy Plus, como motor de cálculo de la simulación, con el apoyo del simulador Design Builder, como interfase gráfica.

Fueron necesarias estrategias para calibrar las simulaciones realizadas, pues, por un lado, los métodos de medición utilizados no podían determinar aspectos tales como:

- Hermeticidad o Infiltración del edificio - Perfil de funcionamiento de la ventilación - Energía Residual en el edificio

-Perfiles de utilización de abertura de ventanas y cortinas.

Y, por otro, debíamos tener en cuenta estrategias ligadas a la demanda considerando únicamente el comportamiento pasivo del edificio.

Una vez monitorizado el edificio existente y calibrado su resultado se obtuvieron las demandas de refrigeración y calefacción y fueron la base de un estudio de las mejoras posibles, considerando una realidad que era objeto del proyecto:

\section{RESULTADOS DE CARGAS DEL EDIFICIO EXISTENTE}

Gráficos 4 y 5.

\section{RESUMEN DE LA DEMANDA TÉRMICA DEL EDIFICIO EXISTENTE}

Demanda en refrigeración de las oficinas del ITL: $24.652,36$ kWh

Demanda en calefacción de las oficinas del ILT: 14.610,05 kWh

Total de la demanda Térmica: $39.262,41$ kWh (consumo año)

Indicador de la demanda: $62 \mathrm{kWh} / \mathrm{m}^{2}$ año

\section{COMPROBACIÓN DE AHORRO DESDE LAS ESTRATEGIAS TOMADAS EN PROYECTO}

1.- Colocación de aislamiento en fachadas exteriores 
La incorporación de una nueva capa de aislante de $6 \mathrm{~cm}$ en los muros exteriores, consigue una reducción del $26 \%$ de pérdida interior-exterior en fachada y una reducción del $12 \%$ considerado el volumen total de edificio (fachada -cubiertas- suelo), según los estudios realizados por BeeGroup-CIMNE. El ahorro total del consumo térmico (por demanda con aislamiento de calefacción y refrigeración) representa una ahorro porcentual del $7 \%$. Cabe destacar, en la comprobación numérica, que la opción de instalar el aislante en la parte más exterior del muro favorece una inercia positiva para las cargas de calefacción y refrigeración del edificio.

2.- Colocación de lamas móviles en las fachadas

Las lamas consiguen disminuir las ganancias solares con la consecuente reducción de la demanda de refrigeración. Así pues con lamas el ahorro en refrigeración es de $6.324,07 \mathrm{kWh}$, pero aumentamos las cargas de calefacción en 5.023,08 kWh. El ahorro total de consumo térmico con lamas es de $1.300,99 \mathrm{kWh}$, que representa un ahorro porcentual del $2 \%$ del consumo total de edificio.

\section{Datos concluyentes de las simulaciones ITL-BeeGroupCIMNE}

Con las medidas que se han podido implantar en el simulador calibrado los resultados son los siguientes (no olvidemos que los datos únicamente se refieren a una oficina):

\section{Edificio existente}

Demanda en calefacción: 14.610,05 kWh Demanda en refrigeración: 24.652,36 kWh Total demanda Térmica: 39.262,41 kWh (consumo año)

\section{Edificio Lamas + Aislamiento $(6 \mathrm{~cm})$}

Demanda calefacción: 23.394,47 kWh Demanda refrigeración: 10.030,33 kWh Demanda térmica total: 33.424,80 kWh

\section{Ahorro global 15\%}

El estudio liderado por BeeGroup-CIMNE y su punto de partida, desde la monitorización del edificio existente, no pudo contemplar las aportaciones en el confort de los invernaderos-umbráculos, simplemente se obtuvieron los datos de reducción de la demanda desde las decisiones de aislamiento y protección solar. El estudio desarrollado con Societat Orgánica fue la guía de las decisiones de proyecto.
Gráfico 4

Demanda de refrigeración. Perfil mensual de cargas de refrigeración.

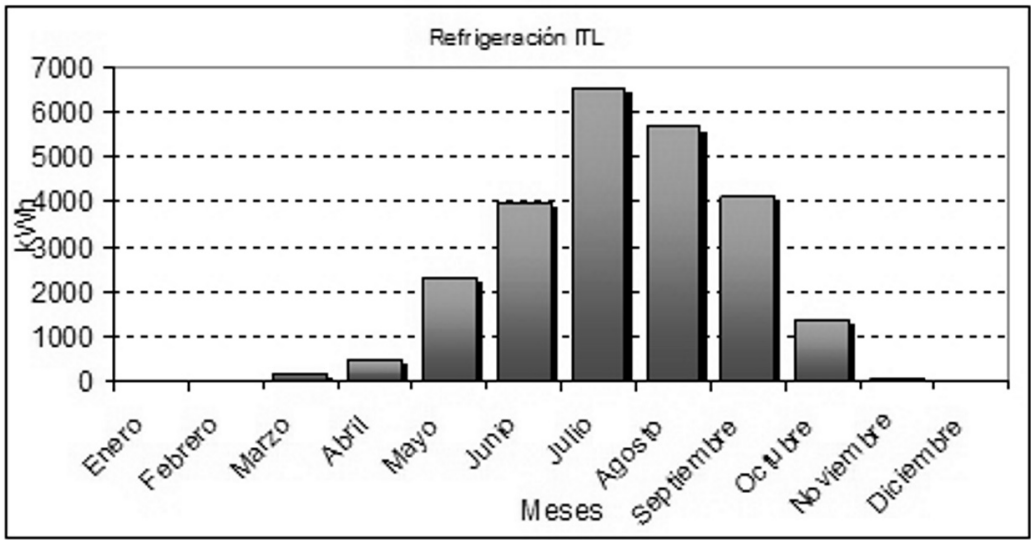

Gráfico 5

Demanda de calefacción. Perfil mensual de cargas de calefacción.

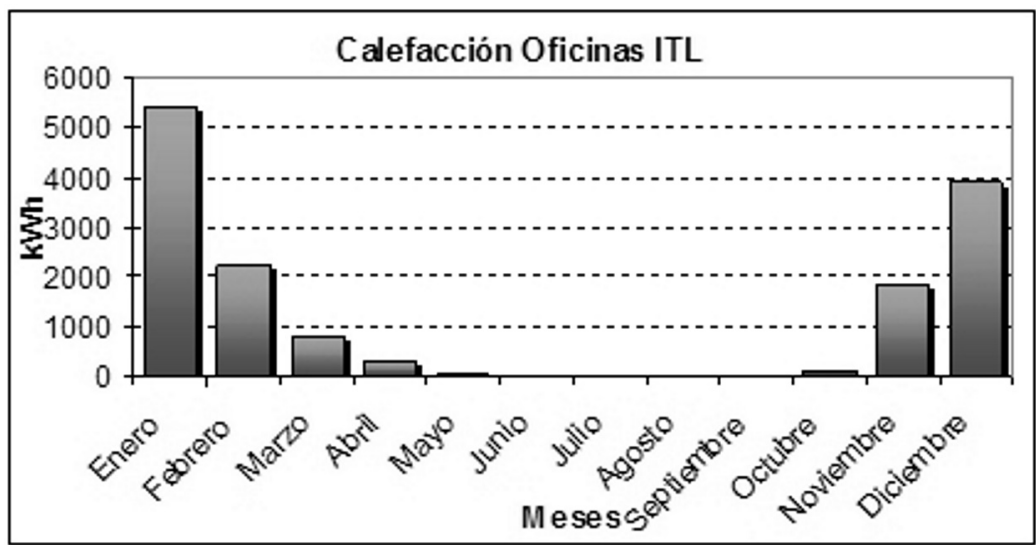

La posibilidad de poder monitorizar el edificio existente -que ITL, BeeGroup y la Universidad de Lérida nos brindó-, ratificaron y dieron fiabilidad a las posibilidades de ahorro energético desde decisiones bioclimáticas. La colaboración con el equipo liderado por BeeGroup-CIMNE, su estudio pormenorizado del edificio y su implicación en fase de obra, ha posibilitado poderlos implicar en el seguimiento del buen funcionamiento de los sistemas de programación y automatización de los invernaderos-umbráculos utilizados.

BeeGroup-CIMNE están siendo los responsables en la reprogramación del control automatizado, así como de la monitorización del edificio una vez finalizado y en uso. La posibilidad de integrarlos, con todas sus consecuencias, hace viable un conocimiento a fondo de la realidad.

\section{ESTUDIOS DE MONITORIZACIÓN DEL EDIFICIO YA CONSTRUIDO. ESTRATEGIA Y SEGUIMIENTO DE BeeGroup-CIMNE (trabajo en curso)}

Se monitoriza el impacto de los invernaderos en el consumo energético del conjunto del edificio, la estrategia de monitorización 
se basa en el control y acciones necesarias para conseguir un confort térmico en los espacios intermedios y en el interior de los edificios.

Se analiza con sensores de control la transferencia de calor de los muros en contacto con el espacio invernadero y el nivel de estratificación del aire en los espacios invernadero.

Para ello se monitoriza con el software de simulación dinámica (Energy Plus), éste nos permitirá cuantificar el impacto energético del invernadero sobre el edificio desde los aspectos de confort (radiación, temperaturas obtenidas en el espacio invernadero y humedad relativa).

Los resultados que se obtendrán del estudio serán:

-Evolución anual del impacto del invernadero sobre el edificio.

- Variación del confort en el interior del invernadero.

-Evaluación del sistema de regulación del invernadero.

\section{Estrategias bioclimáticas de control}

Por un lado, se definen los dispositivos necesarios (control automatizado) para el buen comportamiento de los invernaderos. Es interesante detallar que los invernaderos reciclados ya tenían sus sistemas de control (sistemas de control térmico para necesidades agrícolas), BeeGroup-CIMNE debe reprogramar y ajustar los sistemas a las necesidades propias de un edificio terciario.

Por otro, se trazan las estrategias de análisis del correcto comportamiento térmico de los espacios, el trabajo de campo trata de estudiar los dos invernaderos centrales (de igual tamaño, por tanto con un comportamiento térmico idéntico) y trazar una motorización distinta en cada invernadero que permita extraer datos del impacto del espacio sobre la ventilación, el sombreado y la refrigeración evaporativa.

\section{Descripción de los sistemas de control}

Los sistemas de control del clima del invernadero se resumen en:

1. Sistema motorizado de apertura de los huecos de la cubierta acristalada.

2. Sistema motorizado de apertura de los huecos de cierre de fachada (aperturas NO y SE).

3. Sistema de protección solar mediante pantallas sombreadas.
4. Sistema de humidificación del aire interior del invernadero. Difusores de agua para refrigeración evaporativa.

5. Calibración de las simulaciones con los datos de monitoreo.

6. Simulación de las alternativas.

7. Sistema de control del clima interior del invernadero. Esta gestión del clima se realizará mediante el software ECONOMIC y sondas de radiación y velocidad del aire exterior.

Los dispositivos necesarios para este control son:

- Temperatura ambiente interior de los invernaderos a distintas alturas (colocación de Sonda TESTO).

- Humedad relativa en el interior de los invernaderos a distintas alturas (colocación de Sonda TESTO).

- Temperatura de contacto exterior de las fachadas que dan al interior de los invernaderos (colocación de Sonda TESTO).

- Temperatura ambiente interior de las salas que están en contacto con los invernaderos (colocación de Sonda TESTO).

- Humedad relativa en el interior de las salas que están en contacto con los invernaderos (colocación de Sonda TESTO).

- Temperatura de contacto interior de las paredes de las salas que están en contacto con los invernaderos (colocación de Sonda TESTO).

- Transferencia de calor de las paredes en contacto con el invernadero (colocación de fluxómetro FLUXE).

- Control de las aperturas de los invernaderos en el periodo de estudio.

- Velocidad del aire dentro del invernadero (colocación de anemómetros).

- Puesta en funcionamiento de los aspersores (Control aspersores Control bombea o ECONOMIC software).

- Control aperturas Sonda contacto o ECONOMIC software.

\section{Detalle de la estrategia bioclimática de control del confort}

Se realizarán, en una primera fase, 11 ensayos que darán a conocer el impacto de la ventilación sobre el confort térmico, el impacto del sombreado y la eficiencia de los micro aspersores. Adjuntamos los primeros ensayos que se realizaron en el mes de octubre (actualmente se están analizando los resultados, Gráficos 6, 7 y 8).

\section{CONCLUSIONES}

En los edificios del Parque Científico Tecnológico Agroalimentario estamos en la fase final de un proceso necesario dentro del mundo de la edificación, es imprescin- 
Gráfico 6

Previsión de ensayos para determinar el nivel de ventilación de los invernaderos.

\begin{tabular}{|c|c|c|}
\hline Ensayos: ventilación & Invernadero A & Invernadero B \\
\hline Ventilación 1 & $\begin{array}{l}\text { Aperturas abiertas } \\
\text { Sombreado: } 25 \% \\
\text { Aspersores: OFF }\end{array}$ & $\begin{array}{l}\text { Aperturas cerradas } \\
\text { Sombreado: } 25 \% \\
\text { Aspersores: OFF }\end{array}$ \\
\hline Ventilación 2 & $\begin{array}{l}\text { Aperturas abiertas } \\
\text { Sombreado: } 50 \% \\
\text { Aspersores: OFF }\end{array}$ & $\begin{array}{l}\text { Aperturas cerradas } \\
\text { Sombreado: } 50 \% \\
\text { Aspersores: OFF }\end{array}$ \\
\hline Ventilación 3 & $\begin{array}{l}\text { Aperturas abiertas } \\
\text { Sombreado: } 75 \% \\
\text { Aspersores: OFF }\end{array}$ & $\begin{array}{l}\text { Aperturas cerradas } \\
\text { Sombreado: } 75 \% \\
\text { Aspersores: OFF }\end{array}$ \\
\hline
\end{tabular}

Gráfico 7

Previsión de ensayos para determinar el efecto del sombreado.

\begin{tabular}{|c|c|c|}
\hline Ensayo sombreado & Invernadero A & Invernadero B \\
\hline Sombras 1 & $\begin{array}{l}\text { Aperturas cerradas } \\
\text { Sombreado: } 0 \% \\
\text { Aspersores: OFF }\end{array}$ & $\begin{array}{l}\text { Aperturas cerradas } \\
\text { Sombreado: } 100 \% \\
\text { Aspersores: OFF }\end{array}$ \\
\hline Sombras 2 & $\begin{array}{l}\text { Aperturas cerradas } \\
\text { Sombreado: } 33 \% \\
\text { Aspersores: OFF }\end{array}$ & $\begin{array}{l}\text { A perturas cerradas } \\
\text { Sombreado: } 100 \% \\
\text { Aspersores: OFF }\end{array}$ \\
\hline Sombras 2 & $\begin{array}{l}\text { Aperturas cerradas } \\
\text { Sombreado: } 33 \% \\
\text { Aspersores: OFF }\end{array}$ & $\begin{array}{l}\text { Aperturas cerradas } \\
\text { Sombreado: } 100 \% \\
\text { Aspersores: OFF }\end{array}$ \\
\hline Sombras 3 & $\begin{array}{l}\text { Aperturas cerradas } \\
\text { Sombreado: } 50 \% \\
\text { Aspersores: OFF }\end{array}$ & $\begin{array}{l}\text { Aperturas cerradas } \\
\text { Sombreado:100\% } \\
\text { Aspersores: OFF }\end{array}$ \\
\hline Sombras 4 & $\begin{array}{l}\text { Aperturas cerradas } \\
\text { Sombreado: } 75 \% \\
\text { Aspersores: OFF }\end{array}$ & $\begin{array}{l}\text { Aperturas cerradas } \\
\text { Sombreado: } 100 \% \\
\text { Aspersores: OFF }\end{array}$ \\
\hline
\end{tabular}

Gráfico 8

Previsión de ensayos para determinar la eficiencia de los micro aspersores.

\begin{tabular}{|l|l|l|}
\hline $\begin{array}{l}\text { Ensayo refrigeración } \\
\text { evaporativa }\end{array}$ & \multicolumn{1}{|c|}{ Invernadero A } & \multicolumn{1}{|c|}{ Invernadero B } \\
\hline Aspersión 1 & $\begin{array}{l}\text { Aperturas abiertas } \\
\text { Sombreado: } 10 \% \\
\text { Aspersores: ON, si la } \\
\text { humedad } \leq 20 \%\end{array}$ & $\begin{array}{l}\text { Aperturas Abiertas } \\
\text { Sombreado:10\% } \\
\text { Aspersores: OFF }\end{array}$ \\
\hline Aspersión 2 & $\begin{array}{l}\text { Aperturas abiertas } \\
\text { Sombreado:10\% } \\
\text { Aspersores: OFF }\end{array}$ & $\begin{array}{l}\text { Aperturas abiertas } \\
\text { Sombreado: } 10 \% \\
\text { Aspersores: ON, si la } \\
\text { humedad } \leq 20 \%\end{array}$ \\
\hline Aspersión 3 & $\begin{array}{l}\text { Aperturas abiertas } \\
\text { Sombreado: } 10 \% \\
\text { Aspersores: ON, si la } \\
\text { humedad } \leq 30 \%\end{array}$ & $\begin{array}{l}\text { Aperturas abiertas } \\
\text { Sombreado: } 10 \% \\
\text { Aspersores: OFF }\end{array}$ \\
\hline
\end{tabular}


dible que los arquitectos conozcamos los resultados obtenidos sobre nuestras actuaciones proyectuales, los edificios del Parque Científico Tecnológico Agroalimentario son los primeros edificios en los que podemos profundizar y parametrizar nuestras propuestas bioclimáticas desde la información que obtendremos más allá de los cálculos de proyecto y las opiniones de sus usuarios. El resultado y ajuste que nos proporcionará la motorización y monitorización de los edificios nos da el conocimiento necesario para nuestros futuros proyectos, es necesario analizar las innovaciones que en el proyecto se proponen, así como la complejidad que la realidad supone para seguir trabajando de una forma veraz por la eficiencia energética de nuestros edificios. Como arquitectos somos conscientes que un mal uso de nuestros edificios invalida parte de nuestros esfuerzos de proyecto, más allá del trabajo proyectual es necesario un buen mantenimiento y un buen uso del edificio. Junto con el interés de conocer los resultados reales del edificio se considera clave la información y sensibilización de los usuarios en los criterios de un edificio sostenible.

\section{FICHA TÉCNICA}

Promoción Pública. "Consorcio "PCiTAL" Ayuntamiento de Lérida - Universidad de Lérida". Autores: Felip Pich-Aguilera Baurier (Equip Arquitectura Pich-Aguilera)

José Maria Puigdemasa Hospital

Teresa Batlle Pagés (Equip Arquitectura Pich-Aguilera)

Responsables de grupo: Ángel Sendarrubias, Javier Milanés, Pau Casaldaliga.

Responsable de proyecto: Bernat Ros.

Colaboradores Jordi Camps, Marc Dolcet, Javier Gómez.

Especialistas: SOCIETAT ORGÁNICA, consultores energéticos.

Responsable simulaciones: Fabián López.

Responsable control residuos: Albert Sagrera.

Asesor: Albert Cuchi

Grupo de bioclimatismo y eficiencia energética del Centro de Investigación Cálculos Numéricos (BeeGroup CIMNE). Juntamente con el Instituto Tecnológico de Lleida (ITL) y el Grupo de Energía solar de la Universidad de Lleida. Responsable técnico: Jordi Cipriano.

Felip Solsona, arquitecto técnico.

BOMA-SALA consultores de estructuras. Responsable técnico: Miguel Ángel Sala

EINESA, ingeniería. Técnico responsable: Miquel Gasulla

CLIENTE: Consorci del "Parc Científic i Tecnològic Agroalimentari" (PCiTAL)

CONSTRUCTORA: COMSA

SUPERFICIE: $19.020 \mathrm{M}^{2}$

\section{BIBLIOGRAFÍA}

(1) Warren, P. R. 1977: Ventilation through openings in one wall only. Heat Transfer in Buildings, Proceedings of ICHMT seminar. Hemisphere. New York, EE.UU.

(2) Castilla, N. 1994: Greenhouses in the Mediterranean area: Technological level and strategic management. Acta Horticulturae, 361: 44-56.

(3) Muñoz, P. 1998: Ventilación Natural de Invernaderos Multitúnel. Tesi Doctoral. Escola Técnica Superior de Enginyeria Agrària. Universitat de Lleida.

(4) Oca, J. 199: Estudi de la ventilació natural per efecte tèrmic en hivernacles, mitjançant tècniques de visualització de fluids en models a escala. Tesi Doctoral. Escola Técnica Superior de Enginyeria Agrària. Universitat de Lleida.

(5) Pérez Parra, J. 2002: Ventilación Natural de invernaderos tipo parral. Tesis doctoral. Univ. Córdoba.

(6) Bakker, J. C.; Adams, S. R.; Boulard, T.; Montero, J. I. (2007): Innovative technologies for an efficient use of energy. Acta Horticulturae (en preNsa).

(7) Núñez, M.; Antón, A.; Muñoz, P.; Rieradevall, J. (2007): Incorporació d'eines d'anàlisi ambiental en la producció hortícola. A: ICEA, V Congrés, 1907-2007, cent anys d'agricultura catalana. Castelldefels, 4 - 6 Julio, 2007 (Comunicación). 